\title{
Supervisão de sistemas de Automação de Baixo Custo para a Indústria 4.0
}

\author{
João Paulo Carvalho Henriques*, Antônio Augusto Vilas Boas Teixeira** \\ Egidio Raimundo Neto ${ }^{* * *}$, Alexandre Baratella Lugli *** \\ *Instituto Nacional de Telecomunicações, Santa Rita do Sapucaí MG, 37540-000 \\ Brasil (Tel.: 353471 9300; e-mail: joao.paulo@inatel.br). \\ ** Instituto Nacional de Telecomunicações, Santa Rita do Sapucaí MG, 37540-000 \\ Brasil (Tel.: 353471 9300; e-mail: antonioaugusto@gea.inatel.br). \\ *** Instituto Nacional de Telecomunicações, Santa Rita do Sapucaí MG, 37540-000 \\ Brasil (Tel.: 353471 9300; e-mail: egidio.neto@inatel.br).
}

\begin{abstract}
This paper presents a study of OPC UA protocol applied in Industry 4.0. The history of the protocol and the main features are detailed. An OPC server were embedded in a Raspberry PI to illustrate the application of the protocol in a low-cost supervision system for a level plant without connectivity.
\end{abstract}

Keywords: Supervision System for Automation; OPC UA; Industry 4.0; Industrial Protocol Communication;

Resumo: Este artigo apresenta um estudo sobre o protocolo OPC UA e seu papel na Indústria 4.0. É apresentado um histórico do protocolo e suas principais funcionalidades são detalhadas. Implementou-se um servidor OPC UA utilizando uma Raspberry Pi para ilustrar a aplicação de um sistema de supervisão de baixo custo em uma planta didática de nível que não dispõe de conectividade.

Palavras-chaves: Supervisão de Sistemas de Automação; OPC UA; Indústria 4.0; Protocolos Industriais de Comunicação;

\section{INTRODUÇÃO}

O volume de dados gerado anualmente cresce em ritmo exponencial. Estima-se que em 2025 serão gerados 163 zettabytes de informações mundialmente, dez vezes mais do que em 2016 (International Data Corporation, 2017). O fluxo constante de informações se entranhou de forma permanente à dinâmica do mundo globalizado. Enquanto setores como o mercado financeiro foram revolucionados pela internet, outros apenas agora começam a adotar a conectividade como um pilar fundamental. O setor industrial se enquadra no segundo caso, onde o estado das tecnologias de rede, principalmente no que tange à velocidade e confiabilidade, foi um fator inibidor da adoção em larga escala deste modelo (World Economic Forum, 2015; Wollschlaeger, et al., 2017). A disponibilidade e qualidade de conexões de banda larga estão mudando este panorama. Enquanto a Internet das Coisas vem se tornando o mais importante paradigma no desenvolvimento de produtos voltados ao consumidor final, o setor industrial finalmente começa a beneficiar-se dos dispositivos interconectados com a Internet das Coisas Industrial (IIoT na sigla em inglês).

A IIoT é uma das partes de um processo em andamento que é considerado a Quarta Revolução Industrial, ou Indústria 4.0. Além da maior conectividade, esta revolução provém de dispositivos sensores mais baratos e avanços nos campos de inteligência artificial e machine learning (Schwab, 2016). Esta iniciativa deve alterar toda a estrutura operacional dos empreendimentos industriais, desde o chão de fábrica até o nível gerencial. No ano de 2020 espera-se uma redução de custos de 421 bilhões de dólares anuais decorrentes dos processos modernizados (PWC, 2016).

Esta revolução não consiste apenas na atualização de sensores, atuadores e ferramentas de análise mais poderosas. A comunicação é um ponto crucial de todo este sistema. O novo modelo propõe uma comunicação mais abrangente, com dispositivos inteligentes e uma arquitetura descentralizada. As novas tecnologias que formam a base desta revolução operam com grandes volumes de dados sendo analisados em tempo real, elevando a importância da confiabilidade destes dados a nível vital. Novos protocolos foram e estão sendo desenvolvidos para atender às necessidades das fábricas inteligentes. O objetivo deste trabalho é demonstrar a aplicabilidade do protocolo OPC UA na Indústria 4.0 e propor a implementação prática de um sistema de comunicação baseado nele, a fim de modernizar uma planta que não dispõe de nenhuma comunicação industrial.

Na seção 2 são apresentados os conceitos das tecnologias da Indústria 4.0 e do protocolo OPC UA, incluindo paralelos entre ambos. A seção 3 detalha o desenvolvimento da aplicação prática, descrevendo a arquitetura do sistema e as ferramentas utilizadas. A seção 4 discute brevemente os resultados da aplicação. Por fim, a seção 5 apresenta as conclusões dos autores e sugestões de futuros trabalhos baseados neste. 


\section{CONCEITOS}

\subsection{Indústria 4.0}

O termo Indústria 4.0 (Industrie 4.0) foi cunhado pelo governo alemão para denominar uma iniciativa de modernização do setor industrial do país (World Economic Forum, 2015). Embora esta iniciativa tenha sido concebida especificamente para a Alemanha, as tecnologias que são a base da Indústria 4.0 têm se desenvolvido por todo o mundo e o termo acabou tornando-se genérico para descrever este novo paradigma de produção (Wollschlaeger, et al., 2017). Existem dezenas de outros termos que se referem a estes mesmos desenvolvimentos, como Connected Enterprise e Quarta Revolução Industrial, e embora cada uma destas definições tenha suas particularidades, certos pontos-chaves são comuns a todas.

Fundamentalmente, a Indústria 4.0 refere-se à digitalização em massa dos processos produtivos (Mckinsey, 2015). O barateamento de dispositivos sensores permite que informações sobre o processo que eram até então inacessíveis agora sejam registradas (Schwab, 2016). A conectividade e a Internet das Coisas garantem acesso a dados dos sistemas em tempo real, em qualquer lugar e em qualquer dispositivo. A digitalização remove restrições físicas e transforma operações individuais de uma companhia em um grande sistema integrado.

A Indústria 4.0 deve afetar todos os níveis de operação do setor. O aumento do número de sensores proporciona uma visão a nível micro dos processos previamente inatingível. O maior volume de dados sobre o processo em questão, alimentando o nível de controle e aliado ao aumento da capacidade de processamento deste, permite que ferramentas computacionais analisem seu desempenho em tempo real (Mckinsey, 2015). As ferramentas de machine learning, por exemplo, são algoritmos treinados para realizar uma tarefa e com o tempo tornam-se altamente especializados, aprendendo a partir dos dados com os quais são alimentados (Schwab, 2016). As ferramentas de analytics também trabalham com grandes volumes de dados, a nível gerencial, encontrando tendências e correlações que podem não ser visíveis mesmo em um estudo inicial detalhado do processo, mas que se tornam claras ao longo do tempo. Desta forma os gerentes podem tomar decisões informadas, eliminando gargalos no processo produtivo, diminuindo desperdícios e aumentando sua eficiência. Por fim, a presença das conexões de bandalarga proporciona um ambiente de fábricas conectadas e cientes das necessidades umas das outras. Se uma planta apresenta uma irregularidade, outras podem alterar seu ritmo de produção de forma automática para suprí-la. O sistema opera sob demanda, adaptando-se diretamente às necessidades dos consumidores em um conceito similar a aplicativos como o Uber, em que a interação do usuário determina dinamicamente como recursos serão distribuídos (Schwab, 2016).

Ao contrário da Terceira Revolução Industrial, a Indústria 4.0 propõe uma transição mais gradual e suave. O setor industrial é notavelmente lento para aderir a novas tendências. Normalmente, plantas são projetadas utilizando-se o estado da arte em tecnologia disponível e então mantidas em operação sem grandes alterações pelo maior período de tempo possível. Facilmente encontram-se plantas operando há décadas. Como uma grande parcela das ferramentas da Indústria 4.0 está relacionada a softwares e maior conectividade, apenas uma parcela da infraestrutura deverá ser renovada, facilitando sua adoção no meio industrial. Para efeito de comparação, a Terceira Revolução Industrial substituiu aproximadamente $85 \%$ dos equipamentos presentes na época, enquanto para a Quarta Revolução prevê-se a substituição de apenas $45 \%$ dos equipamentos (Mckinsey, 2015).

Em suma, a Indústria 4.0 descreve cadeias de produção inteligentes, que se adaptam a demandas variáveis desde o nível da matéria-prima até o nível do consumidor final, através de plantas altamente digitalizadas e algoritmos capazes de aprender e otimizar processos complexos a partir de volumes massivos de dados, resultando em processos mais eficientes.

\subsection{Redes Industriais}

Tradicionalmente, o setor industrial é representado por uma pirâmide na qual a base indica os equipamentos que diretamente interagem com os processos como apresentado na Figura 1. A comunicação destes equipamentos com o nível diretamente acima, o controle, é implementada por meio de fieldbuses, ou redes industriais. Os fieldbuses nasceram da necessidade de se simplificar instalações físicas, onde o uso de pares de fios para cada dispositivo tornou-se inviável com o aumento da complexidade dos processos (Thomesse, 2005). As redes industriais oferecem um barramento comum a todos dispositivos compatíveis, além de oferecerem intervalos de comunicação determinísticos e alta confiabilidade.

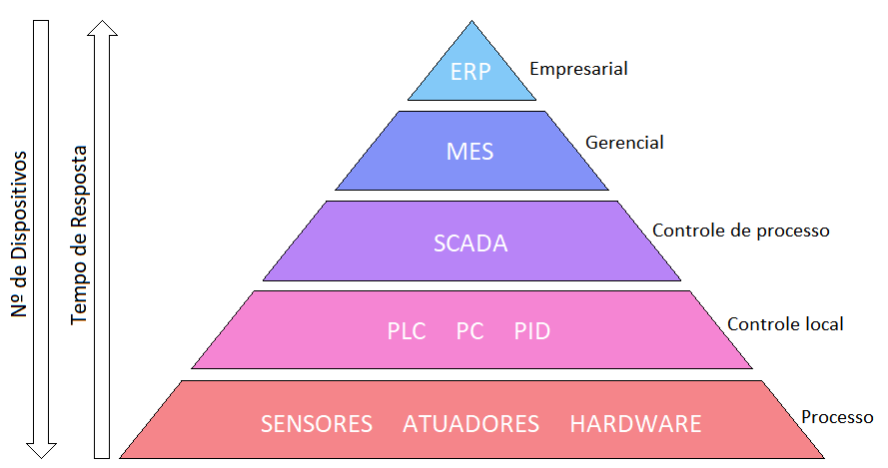

Figura 1 - Pirâmide Representando um sistema de Automação

Atualmente o setor passa por um processo de transição entre tecnologias. Novos paradigmas de produção, como a Indústria 4.0, demandam um volume de informações muito maior do que os fieldbuses clássicos suportam. Tecnologias como ProfiBus e DeviceNet estão sendo substituídas por versões mais novas, como ProfiNet e EthernetIP. Estes novos protocolos compartilham muitas similaridades com o modelo Ethernet, que é pouco utilizado no meio industrial devido a restrições como a baixa imunidade dos pares trançados ou fragilidade da fibra ótica, mas estes atendem também aos requisitos dos ambientes industriais (Dias, et al. 2014). Esta migração alinha-se a paradigmas que visam maior 
conectividade, onde as interfaces entre os níveis da pirâmide tornam-se mais transparentes e os dados podem fluir com maior facilidade.

\subsection{Protocolo OPC}

O protocolo OPC (Open Platform Communications) define uma série de especificações de interfaces de comunicação introduzida em 1996 e projetada inicialmente para solucionar deficiências inerentes a protocolos proprietários da época. A OPC Foundation define OPC como um padrão de interoperabilidade para trocas confiáveis de dados no setor de automação industrial (OPC Foundation, 2018). Um termo chave desta definição foi o motivador para sua concepção: interoperabilidade. Enquanto os fieldbuses estabeleciam uma camada física comum a todos dispositivos em campo, a nível de software cada fabricante possuía sua própria interface. Fabricantes de sistemas SCADA (Supervisory Control and Data Acquisition) e IHM (Interface Homem-Máquina) tinham de criar drivers específicos para cada dispositivo com o qual fossem se comunicar.

Este cenário, apresentado na Figura 2, tornava a configuração de um sistema com equipamentos de vários fabricantes complexo ou até mesmo inviável (Berge, 2005). Em 1995 as empresas Fisher-Rosemount, Intellution, Opto 22 e Rockwell Software se juntaram em uma força-tarefa para solucionar este problema (OPC Foundation, 2018). Em agosto de 1996 foi lançada a primeira especificação do protocolo: OPC Data Access. A medida que o padrão OPC foi se difundindo, novos fabricantes juntaram-se à força-tarefa e a necessidade de uma organização mais rígida e independente tornou-se aparente, resultando na criação da OPC Foundation, a instituição sem fins lucrativos que gerencia o protocolo até hoje. Atualmente, a fundação já conta com mais de 500 empresas afiliadas por todo o mundo (OPC Foundation, 2018).

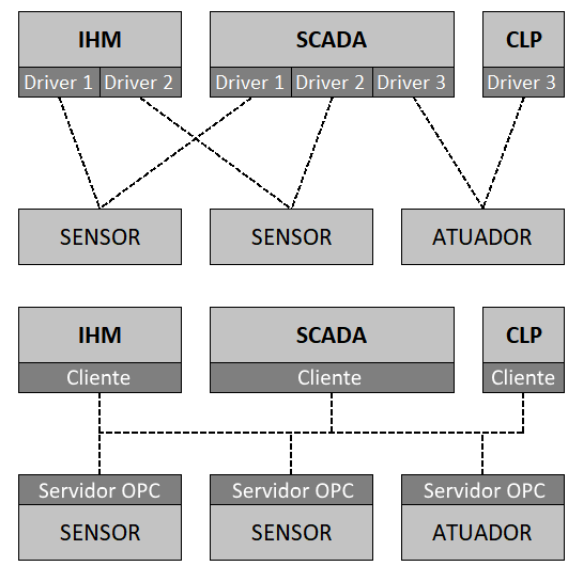

Figura 2 - Comparação sistema "tradicional" e OPC

A ideia por trás do padrão OPC é oferecer uma interface padrão para que os diferentes dispositivos de um sistema se comuniquem sem precisarem entender como funcionam a nível de hardware. O protocolo estabelece operações que todo dispositivo deve implementar e o padrão como os dados devem ser apresentados, mas a forma como estes métodos são implementados fica a critério do fabricante.

OPC DA foi a primeira especificação lançada em 1996 com múltiplas revisões lançadas nos anos seguintes. Até hoje é a especificação implementada no maior número de produtos comerciais diferentes. Ela estabelece a interface para leitura e escrita das variáveis de um processo. Foi concebida para conectar dispositivos de controle como CLPs a dispositivos de visualização como IHMs. O OPC DA estabelece métodos para os clientes se conectarem aos servidores e navegarem o AddressSpace destes. Além disto, todas as variáveis contam com timestamps e um índice da qualidade dos dados (Manhke, et al., 2009).

A especificação OPC A\&E foi o segundo lançamento do protocolo, no ano de 1999. Enquanto a especificação anterior visa o acesso contínuo e cíclico das variáveis do processo, o OPC A\&E atua com eventos. Um evento pode ser definido como a mudança de uma condição no processo que não acontece de forma determinística. Esta especificação estabelece métodos para os clientes serem notificados do acontecimento de eventos, filtrarem os eventos recebidos através de critérios customizáveis e reconhecerem alarmes (Manhke, et al., 2009).

A última especificação do OPC Classic, OPC HDA, foi lançada em 2001. Ela define métodos para interação com bancos de dados históricos. Isto inclui a leitura de dados armazenados, podendo filtrá-los por variável ou timestamp, além da possibilidade de inserir novos dados ou apagar aqueles no servidor (Manhke, et al., 2009).

\subsection{Protocolo OPC UA}

O OPC Classic teve grande aceitação na indústria e ainda é amplamente utilizado, mas suas limitações se tornaram aparentes com o tempo. Embora várias destas limitações tenham sido contornadas com o lançamento de diferentes especificações, a maior delas é irresolúvel: a interface COM/DCOM. A tecnologia proprietária limita o uso do OPC Classic a sistemas Windows, além de estar sendo substituída gradualmente pela Microsoft por outras arquiteturas (OPC Foundation, 2018). A primeira tentativa de lançar uma especificação compatível com vários sistemas operacionais foi o OPC XML-DA que mantém a funcionalidade do OPC DA mas substituí a interface COM/DCOM por HTTP/SOAP. O uso intensivo de recursos e performance limitada fez com que o OPC XML-DA fosse pouco adotado no setor industrial (Manhke, et al., 2009). A necessidade de um protocolo que fosse independente de sistema operacionais e adequado às demandas de processos mais inteligentes resultou na criação do OPC UA.

O OPC Unified Architecture é a mais nova especificação do protocolo OPC, lançada em 2006. Ela vem substituindo a família OPC Classic gradualmente, pois combina todas as funcionalidades anteriores (DA, A\&E e HDA) em um único protocolo (OPC Foundation, 2018). O OPC UA oferece um 
grau de flexibilidade maior que seus antecessores. Além de ser independente da plataforma de execução, ele oferece uma comunicação mais eficiente e permite expor um modelo de informações mais completo, sendo ideal para modelar sistemas complexos. É também totalmente escalável, podendo ser executado com o mínimo de funcionalidades necessárias em um sistema embarcado ou com métodos sofisticados em um grande servidor. O OPC UA apoia-se em dois pilares fundamentais: transporte de dados e modelagem da informação (Manhke, et al., 2009).

Cada servidor OPC UA possui um AdressSpace associado a si. Este é definido como o conjunto de informações que os clientes podem acessar (OPC Foundation, 2018). No OPC Classic os dados que podem ser acessados são limitados. São oferecidos o valor de uma variável, seu nome e unidade de engenharia. Os objetos do AdressSpace UA oferecem um modelo completo com metadados em conjunto com as variáveis. Metadados são informações sobre outras informações. Por exemplo: um sensor de pressão operando com OPC Classic vai apenas retornar um valor numérico correspondente à sua leitura quando inquirido por um cliente. Este sensor, operando com OPC UA, pode retornar além de sua leitura: seu modelo, a unidade da leitura, sua tolerância e uma infinidade de outras informações que podem ser customizadas de acordo com a aplicação. Estes metadados potencializam a aplicação de ferramentas de machine learning e analytics. Suponha, por exemplo, que o sensor comece a apresentar leituras errôneas. De posse de metadados como o modelo do sensor em questão, os algoritmos de controle podem detectar a falha e traçar um perfil comportamental deste dispositivo com o tempo. Se outro sensor no futuro começar a apresentar um comportamento similar ao componente defeituoso, os algoritmos podem solicitar uma manutenção de forma totalmente automática, reduzindo custos operacionais e tempo ocioso da linha de produção.

Além da presença de metadados, a forma como o AdressSpace pode ser organizada é o que possibilita ao OPC UA oferecer uma modelagem completa de um sistema. O AdressSpace é composto por nodes, que possuem atributos e referências. Os atributos são os dados e metadados que este node armazena e as referências são ligações entre os nodes (OPC Foundation, 2006) (Manhke, et al., 2009).

O OPC UA utiliza conceitos de orientação a objetos em sua abordagem (OPC Foundation, 2006). Técnicas como herança e classes permitem que objetos, traçando um paralelo com técnicas de programação, sejam criados, agilizando o processo de implementação de um sistema. A Figura 3 apresenta um exemplo de como um motor pode ser modelado e inserido no sistema como um objeto. Uma biblioteca de modelos similares pode ser disponibilizada por fabricantes e customizada pelos consumidores, simplificando significativamente o processo de configuração do AdressSpace.

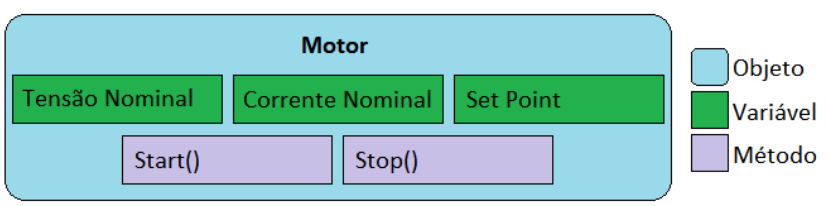

Figura 3 - Exemplo do uso de classes

Além da modelagem de dados, o OPC UA enfatiza o transporte destes dados. São definidos vários métodos de codificação, segurança e transporte diferentes, permitindo ao usuário escolher a combinação que o atenda melhor em uma análise de custo e performance (Manhke, et al., 2009). A Figura 4 ilustra o stack de comunicação do protocolo.

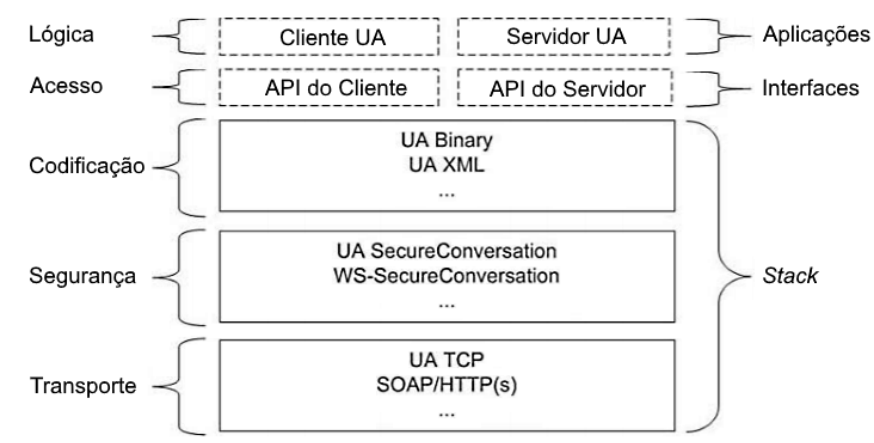

Figura 4 - Stack do Protocolo OPC UA (Manhke, et al., 2009).

Em aplicações que dispõem de baixo poder de processamento, ou em que o tempo de resposta é crucial, como em um servidor embarcado em um dispositivo no chão de fábrica, o stack pode ser configurado para utilizar os métodos nativos: UA Binary, UA SecureConversation e UA TCP. Nesta configuração as variáveis são transmitidas com pouco tratamento adicional, acrescentando-se apenas identificadores que informam seu tipo. Estes dados "crus" garantem o menor overhead de processamento possível, em troca da compatibilidade limitada a outras aplicações OPC. Já em uma situação em que os dados serão consumidos por múltiplas aplicações que não necessariamente utilizam OPC, como em um software gerencial de uma companhia, pode-se utilizar um stack orientado a serviços web, que apresenta maior compatibilidade com firewalls além de codificar os dados no padrão XML, que é utilizado por inúmeras especificações (Manhke, et al., 2009).

Embora a plataforma UA apresente mudanças significativas em relação às suas antecessoras, isto não torna todos os produtos OPC Classic automaticamente obsoletos. O OPC UA foi projetado de forma que possa ser traduzido de maneira simples para as outras especificações e vice-versa. Esta compatibilidade entre especificações permite uma implementação gradual do OPC UA, inicialmente sem a necessidade da troca de nenhum dispositivo, apenas a adição das interfaces tradutoras. A medida que os dispositivos forem substituídos por modelos mais modernos é possível beneficiarse da gama completa de ferramentas do OPC UA (Manhke, et al., 2009). 
Todas estas funcionalidades apresentadas são apenas uma pequena parcela do que o OPC UA oferece, mas demonstram porque este protocolo é uma escolha ideal para a Indústria 4.0.

\section{APLICAÇÃO PRÁTICA}

A aplicação prática proposta neste trabalho consiste na implementação de um servidor OPC UA em uma planta que não dispõe de nenhum meio de comunicação industrial. Deseja-se demonstrar como uma aplicação isolada pode ser facilmente convertida a um dispositivo IIoT com uma solução de baixo custo. $O$ ponto de partida foi determinar a arquitetura geral do sistema, ilustrada na Figura 5. Esta foi concebida visando expor algumas das características do protocolo OPC apresentadas na seção anterior, como a compatibilidade com vários sistemas operacionais e a escalabilidade do protocolo.

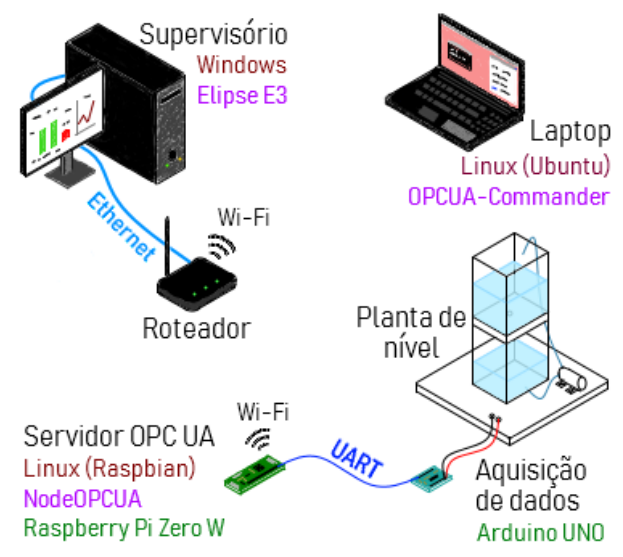

Figura 5 - Arquitetura da Aplicação

A operação do sistema pode ser descrita de forma resumida. A planta representa o processo industrial, que possuí variáveis físicas que o caracterizam. Estas variáveis são digitalizadas pela etapa de aquisição de dados e convertidas para um padrão compatível com o protocolo OPC. O servidor disponibiliza estes dados em seu AddresSpace. A etapa de transmissão gerencia a forma como as informações trafegam fisicamente entre servidor e clientes. Por fim, o supervisório e o laptop solicitam os dados do servidor e os apresentam em uma interface visual. A seguir são detalhadas as etapas do sistema, explicando seu funcionamento e a motivação por trás de decisões tomadas no processo de desenvolvimento.

\subsection{Planta de Nível}

A planta utilizada, apresentada na Figura 6 , é um sistema de controle do nível de uma coluna de água projetado para fins acadêmicos. Ela dispõe de dois tanques empilhados, onde o inferior atua como reservatório, armazenando a água do sistema em repouso, e o superior é onde o nível é controlado, sendo aferido por um sensor ultrassônico instalado em seu topo. Uma bomba hidráulica conecta os dois tanques, e uma abertura no fundo do tanque superior garante escoamento de água contínuo para o inferior. A potência da bomba é ajustada proporcionalmente à sua alimentação, que varia de $0 \mathrm{a} 10 \mathrm{~V}_{\mathrm{DC}}$.
$\mathrm{O}$ sensor fornece uma tensão de saída de 0 a $3,3 \quad V_{D C}$ proporcional ao nível medido no tanque superior.

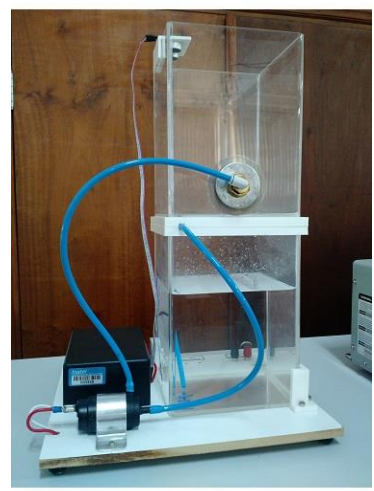

Figura 6 - Planta de Nível

Para esta aplicação são monitorados: o volume e nível das colunas de água nos tanques e a potência da bomba

\subsection{Sistema de Aquisição de Dados}

A etapa de aquisição de dados amostra as tensões de interesse na planta e as converte a valores digitais. Inicialmente, esta etapa seria executada na mesma plataforma do servidor OPC UA, mas devido a uma limitação do hardware escolhido para executar o servidor (ausência de conversores analógico/digitais) estas fases tiveram de ser desacopladas e executadas em plataformas separadas. A aquisição de dados foi então implementada em um Arduino UNO, escolhido por sua acessibilidade e alta disponibilidade no mercado.

Os conversores analógico/digitais do Arduino possuem resolução de 10 bits e a tensão de referência utilizada foi de 5 $\mathrm{V}_{\mathrm{DC}}$. São utilizados dois conversores conectados ao sensor da planta e à alimentação da bomba, como apresentado na Figura 7. Para esta segunda conexão é possível observar que um divisor de tensão foi utilizado. Sua função é adequar o nível da tensão de entrada ao conversor, que comporta até 5 VDC. O valor dos resistores é sugerido pelo manual do ATmega328P, o microcontrolador utilizado no Arduino UNO (ATMEL Corporation, 2016).

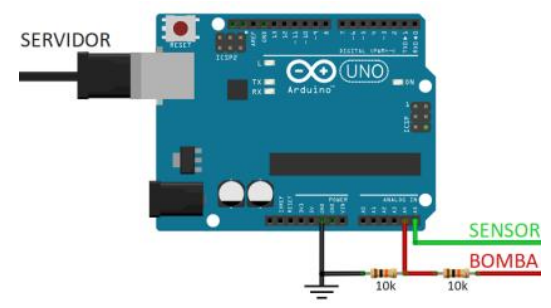

Figura 7 - Arduino para Aquisição de Dados

Com as variáveis da planta digitalizadas, seus valores são convertidos da escala de 10 bits a unidades que expressam as grandezas medidas no sistema internacional. Por fim, as variáveis são enviadas para o microcontrolador que executa o servidor através de uma conexão UART (Universal Asynchronous Receiver/Transmitter) entre as portas USB das plataformas. A taxa de transmissão escolhida foi de 9600 bits 
por segundo por se tratar de um volume relativamente baixo de dados. Os valores são transmitidos em uma string, ilustrada na Figura 8, que possui caracteres fixos a fim de demarcar o início e fim de cada valor numérico e qual o significado deste.

\section{P86PU62UL73Lu1395ul1642l}

$$
\begin{aligned}
& \text { - Caracteres de controle } \\
& \text { - Potência da bomba [\%] } \\
& \text { - Nível superior [mm] } \\
& \text { - Nível inferior [mm] } \\
& \text { - Volume superior [mL] } \\
& \text { - Volume inferior [mL] }
\end{aligned}
$$

Figura 8 - String de Dados enviadas ao Servidor

\subsection{Servidor OPC UA}

O servidor OPC UA disponibiliza os dados da planta de nível para acesso pelos clientes. O primeiro passo foi determinar a API (Application Programming Interface) que seria utilizada em sua implementação. Para este trabalho optou-se por uma alternativa open-source, o NodeOPCUA. Esta API implementa todo o stack OPC via JavaScript. Determinada a API, o próximo passo foi escolher uma plataforma compatível. Sendo o componente principal do sistema, sua especificação teve de atender alguns requisitos do protocolo e da aplicação. Primeiramente, o servidor devia ser executado em uma plataforma embarcada, alinhando-se ao conceito de dispositivos IIoT e o de um sistema que pode ser acoplado a uma planta já funcional. Além disto, a plataforma utilizada deveria ser capaz de conectar-se à uma rede local, preferencialmente via Wi-Fi. Foi escolhida a Raspberry $P i$ Zero W. A plataforma possui o grande diferencial de poder trabalhar com um SO (Sistema Operacional) baseado em Linux (Raspbian). O sistema Linux conta com amplo suporte e recursos disponíveis, além de suportar a API necessitando poucas configurações adicionais. A presença de um SO também provê uma camada de abstração entre o hardware e as aplicações de alto nível e implementa todo o stack de comunicação $\mathrm{Wi}-\mathrm{Fi}$ nativamente, agilizando o processo de desenvolvimento.

O algoritmo executa o processo de inicialização do servidor e configura seu AddressSpace adicionando as variáveis relativas à planta. Quando pacotes são recebidos via UART os valores são processados e atualizados. A Raspberry fica conectada a uma rede Wi-Fi local, onde todos os dispositivos conectados podem acessar o servidor.

\subsection{Transmissão de Dados}

A etapa de transmissão consiste apenas de um roteador, cuja função é manter uma rede local em que os dispositivos possam interagir. Todos dispositivos trabalharam com endereços IP (Internet Protocol) dinâmicos, atribuídos automaticamente pelo servidor DHCP (Dynamic Host Configuration Protocol) do roteador. Não é necessária nenhuma outra funcionalidade extra deste. O único requisito de hardware é que o roteador deve suportar uma das especificações Wi-Fi 802.11 b/g/n, que são as únicas compatíveis com a Raspberry utilizada. Neste sistema foi utilizado um D-Link DIR-610.

$\mathrm{O}$ roteador conecta-se à Raspberry e ao laptop via Wi-Fi, enquanto a conexão com o supervisório se dá via Ethernet. Esta diferença foi intencional com o propósito de reforçar que o protocolo OPC trata de interações entre aplicações a nível de software, independente do meio físico.

\subsection{Servidor OPC UA}

O supervisório é um cliente OPC que apresenta os dados da planta em tempo real através de uma interface gráfica que representa o processo. A aplicação foi criada com o Elipse E3, uma ferramenta para monitoramento de processos para sistemas Windows. O Elipse pode ser executado em modo de demonstração com todos seus recursos habilitados, incluindo drivers de comunicação OPC UA. A interface desenvolvida é apresentada na Figura 9.

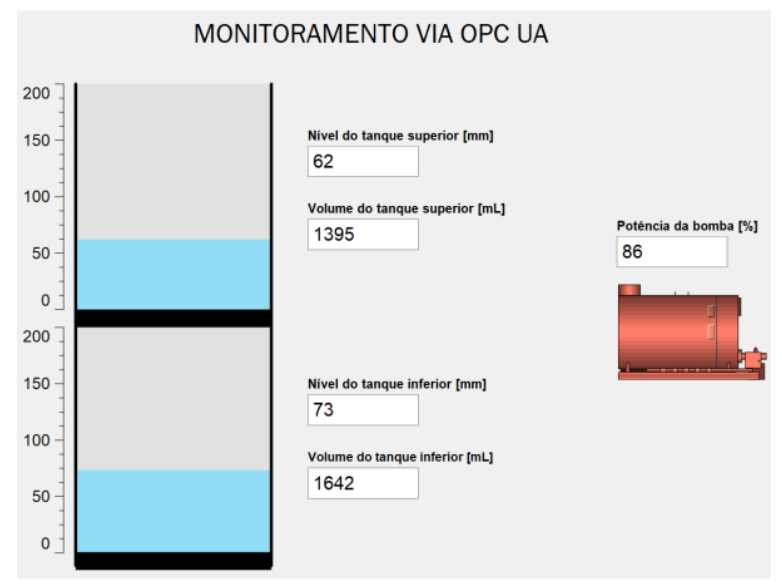

Figura 9 - Interface Gráfica do Supervisório

\subsection{Segundo Cliente OPC (Laptop)}

O laptop executa um segundo cliente OPC em adição ao supervisório. Sua função é demonstrar como um dispositivo móvel pode conectar-se um servidor OPC facilmente e também como o OPC UA pode ser utilizado em diversos sistemas operacionais.

O laptop utiliza o SO Ubuntu, baseado em Linux. O cliente utilizado foi o opcua-commander, uma aplicação em JavaScript baseada na API NodeOPCUA. Não foi criada nenhuma interface visual que representa a planta nesta etapa, mas o cliente conta com uma interface própria, retratada na Figura 10, que o permite navegar pelo AddressSpace e monitorar as variáveis da planta. 


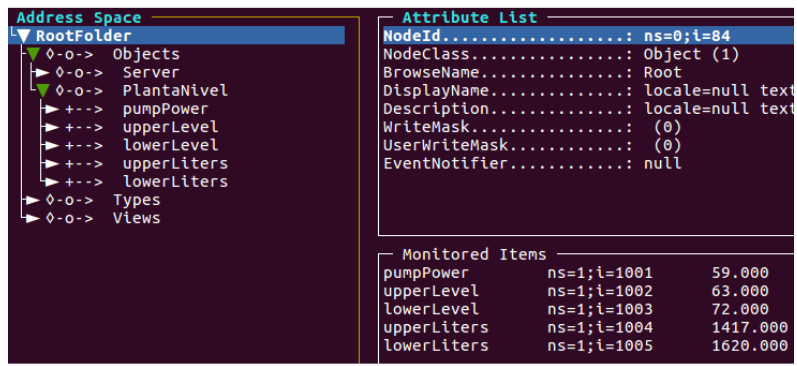

Figura 10 - Interface oрсиа-commander

\section{RESULTADOS}

O sistema final funcionou de acordo com o esperado. A proposta era converter uma planta sem comunicação em um dispositivo IIoT e o objetivo foi atingido. Os testes de funcionamento foram realizados em um ambiente de laboratório no qual a distância máxima entre os dispositivos e roteador foi de aproximadamente 1 metro e não haviam obstáculos entre eles. Não foi utilizado nenhum sistema de controle dinâmico na planta de nível, com a potência da bomba sendo regulada manualmente apor meio de uma fonte de tensão. A Figura 11 apresenta a montagem do sistema final em funcionamento.

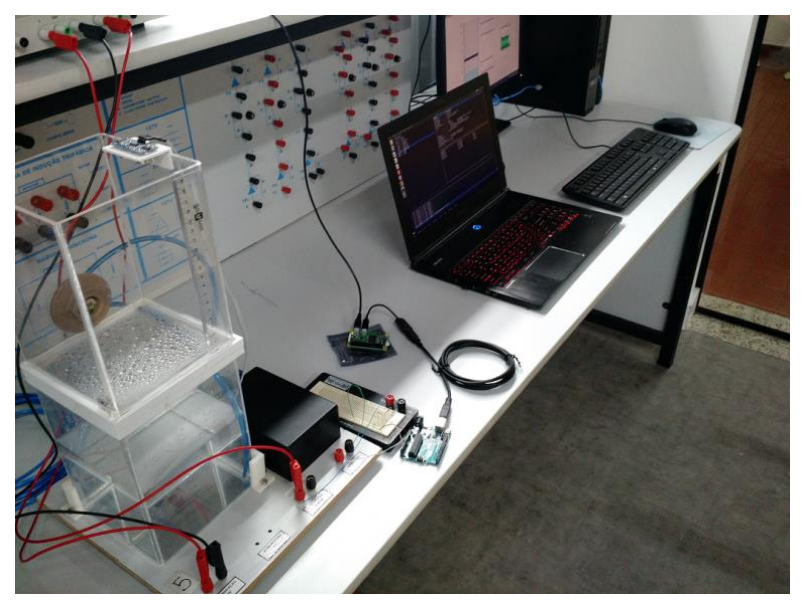

Figura 11 - Montagem Final do Sistema de Supervisão

\section{CONCLUSÕES}

Pode-se perceber o crescimento exponencial da quantidade de dispositivos inteligentes no cotidiano. A conectividade tornouse um requisito essencial em qualquer aplicação moderna e ao longo deste trabalho demonstrou-se que o setor industrial não é diferente. Iniciativas como a Indústria 4.0 prometem mudanças significativas na forma como processos produtivos serão encarados no futuro. O estudo aqui realizado sobre o protocolo OPC UA comprovou que este é uma alternativa ideal para a comunicação destes novos sistemas. Pontos como sua segurança e stack de comunicação altamente configurável são absolutamente necessários em sistemas que trabalharão de forma integrada à internet. $\mathrm{O}$ fato de ser um protocolo totalmente aberto e também suportado por grandes fabricantes assegura a presença do OPC UA no meio industrial no futuro. O OPC UA também atende à proposta de uma transição suave entre tecnologias da Indústria 4.0, com a própria aplicação prática aqui apresentada demonstrando como um sistema já existente pode ser modernizado.

O sistema implementado funcionou perfeitamente e permitiu demonstrar algumas características do OPC UA em uma aplicação real. Entretanto, algumas ressalvas têm de ser feitas. Primeiramente, é preciso destacar que o sistema está extremamente superdimensionado para a aplicação em questão. Um único servidor com poucas variáveis utiliza uma fração minúscula do processamento de uma plataforma como a Raspberry. O Arduino também foi escolhido apenas por seus conversores AD integrados, podendo-se utilizar outra solução mais simples em seu lugar. Idealmente as duas etapas seriam executadas em um único microcontrolador especificado para a aplicação.

O processo de escolha destes componentes foi orientado pela facilidade que eles proporcionariam no processo de implementação do sistema. Com componentes superdimensionados pode-se deduzir que o sistema não apresenta o melhor custo-benefício possível. Embora a aplicação apresente um valor baixo para o setor industrial, não necessariamente este valor reflete a realidade de um produto destinado ao consumidor final. Também é importante destacar que a aplicação foi desenvolvida para operar em um meio acadêmico e de forma alguma está preparada para um ambiente industrial real. Os componentes de hardware e software utilizados não atendem os diversos requisitos de segurança e performance deste tipo de ambiente.

Por fim, para trabalhos futuros, sugere-se o estudo dos impactos que a implementação de uma comunicação compatível com a Indústria 4.0 causam na produtividade de uma planta. Ferramentas de inteligência artificial e machine learning podem ter seus resultados comparados com técnicas de controle tradicionais.

\section{REFERÊNCIAS}

ATMEL Corporation, 2016. ATmega328/P Datasheet. s.1.:s.n. Berge, J., 2005. Software for Automation: Architecture, Integration, and Security. s.1.:ISA.

Dias, A., 2014. Análise comparativa de desempenho de redes Profibus DP e Profinet. Belo Horizonte, s.n., pp. 24302437.

International Data Corporation, 2017. Data Age 2025: The Evolution of Data to Life-Critical, Framingham, Estados Unidos: s.n.

Manhke, W., Leitner, S. \& Damm, M., 2009. OPC Unified Architecture. Nova Iorque: Springer.

MANHKE, W., LEITNER, S. \& DAMM, M., 2009. OPC Unified Architecture. Nova Iorque: Springer.

Mckinsey, 2015. Industry 4.0: How to navigate digitization of the manufacturing sector, s.l.: s.n.

OPC Foundation, 2006. OPC Unified Architecture Specification Part 1: Concepts. s.1.:s.n. 
OPC Foundation, 2006. OPC Unified Architecture Specification Part 3: AdressSpace. s.l.:s.n.

OPC Foundation, 2018.

[Online] Available at: https://opcfoundation.org/about/what-isopc/

OPC Foundation, 2018. [Online] Available at: https://opcfoundation.org/about/opcfoundation/history/

OPC Foundation, 2018. [Online] Available at: https://opcfoundation.org/members

OPC Foundation, 2018. [Online] Available at: https://opcfoundation.org/about/opctechnologies/opc-ua/

OPC Foundation, 2018. OPC Classic. [Online] Available at: https://opcfoundation.org/about/opctechnologies/opc-classic/

PWC, 2016. Industry 4.0: Building the digital enterprise, s.1.: s.n.

Schwab, K., 2016. The Fourth Industrial Revolution. Geneva: World Economic Forum.

Thomesse, J., 2005. Fieldbus technology in industrial automation. Procedings of the IEEE, 93(6), pp. 10731101.

Wollschlaeger, M., Sauter, T. \& Jasperneite, J., 2017. The future of industrial communication: Automation networks in the era of the internet of things and industry 4.0. IEEE Industrial Electronics Magazine, 11(1), pp. 17-27.

World Economic Forum, 2015. Industrial Internet of Things: Unleashing the Potential of Connected Products and Services, Geneva, Suíça. 